\title{
Memory performances and personality traits in mothers of children with obstructive sleep apnea syndrome
}

This article was published in the following Dove Press journal: Psychology Research and Behavior Management

\author{
Daniela Smirni $\mathbb{D}^{1, *}$ \\ Marco Carotenuto ${ }^{2, *}$ \\ Francesco Precenzano 2 \\ Pietro Smirni ${ }^{3}$ \\ Francesca Felicia Operto 4 \\ Rosa Marotta ${ }^{5}$ \\ Michele Roccella' \\ 'Department of Psychology, Educational \\ Science and Human Movement, \\ University of Palermo, Palermo, Italy; \\ ${ }^{2}$ Clinic of Child and Adolescent \\ Neuropsychiatry, Department of Mental \\ Health, Physical and Preventive Medicine, \\ Università Degli Studi Della Campania \\ "Luigi Vanvitelli", Naples, Italy; \\ ${ }^{3}$ Department of Educational Sciences, \\ University of Catania, Catania, Italy; \\ ${ }^{4}$ Child Neuropsychiatry Unit, \\ Department of Basic Medical Sciences, \\ Neuroscience and Sense Organs, \\ University of Bari Aldo Moro, Bari, Italy; \\ ${ }^{5}$ Department of Health Sciences, \\ University "Magna Graecia”, Catanzaro, \\ Italy
}

*These authors contributed equally to this work
Background: Chronic diseases in pediatric age have been identified as stressful risk factors for parents. Studies on caregivers have documented the impact of chronic parenting stress on emotion and cognition.

Aim: To investigate the differences between a group of mothers of children affected by obstructive sleep apnea syndrome (OSAS) for at least 4 years and a group of mothers of typically developing children (TDC) in relation to parental stress, self-esteem, locus of control, and memory performances.

Methods: A group of 86 mothers (mean age 35.6 \pm 4.9 , ranged between 32 and 41 years) of

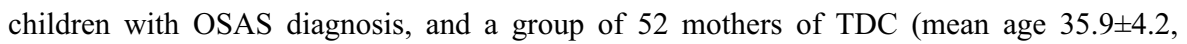
ranged between 32 and 41 years) participated in the study. All participants were administered stress level, global self-esteem, internal/external locus of control scales, and memory assessment.

Results: Mothers of OSAS children, compared to mothers of TDC, had a significantly higher level of stress, lower self-esteem, more external locus of control and poorer memory performance.

Conclusions: The child respiratory disease, with its sudden and unpredictable features, appeared as a significant source of stress for the mother. Such stress condition may have an impact on mothers' personality traits (self-esteem, locus of control) and on their memory performances. The data have suggested a need for psychological support programs for mothers to better manage stress associated with children's respiratory disease.

Keywords: parental stress, memory performances, self-esteem, locus of control

\section{Introduction}

Chronic diseases in pediatric age, including respiratory problems, have been identified as stressful risk factors for parents. ${ }^{1}$ Obstructive sleep apnea syndrome (OSAS) in children is a disorder of breathing during sleep characterized by repeated, temporary episodes of partial or complete upper airway obstruction, disrupting nocturnal ventilation and sleep patterns. ${ }^{2}$ Two features of this childhood respiratory disease seem to be crucial: unpredictability and comorbidity. Crisis occurs during sleep in a sudden, unpredictable, uncontrollable. Moreover, OSAS may be associated with several comorbidities. $^{2}$ Neurocognitive and cardiovascular complications, obesity, daytime sleepiness, hypertension, impaired growth, and learning and behavioral problems, ${ }^{3-6}$ as well as attention, executive functions, and memory difficulties have been documented in children with OSAS. ${ }^{7-10}$ Abnormal neuronal metabolites were detected in the
Correspondence: Daniela Smirni Department of Psychology, Educational Science and Human Movement, University of Palermo, viale delle Scienze, Ed. I5, Palermo 90128 , Italy

Tel +3909123897748

Email daniela.smirni@unipa.it 
hippocampus and frontal cortex of OSAS children. ${ }^{11}$ It has been hypothesized that untreated childhood OSAS could permanently impact on the cognitive development. In addition, a higher incidence of mortality, compared to controls, was found. ${ }^{3}$

OSAS in children appears as a complex pathological condition. Especially since the interruption of breathing can be easily associated with the idea of sudden death. Therefore, its management can be a highly stressful event and become particularly demanding for the family. ${ }^{12-14}$

Nevertheless, to the best of our knowledge, there are few studies on the impact of childhood OSAS on parenting stress. However, several studies on parents of children with chronic or degenerative diseases have focused on the effects of parental stress in cognitive and emotional functioning ${ }^{15,16}$ showing that parenting a child with developmental problems, or a pathological condition, can be a significant source of parental stress. ${ }^{17-21}$

According to the learned helplessness stress model, OSAS can be viewed as an uncontrollable and unpredictable aversive event. ${ }^{22}$ Any respiratory crisis can become an "independent" event that occurs outside of parent's control who experience every time a high helplessness' condition. ${ }^{23}$ At the occurrence of every crisis, the mother still lives another stressful experience of helplessness that can decrease self-confidence in coping with stressors. A positive self-concept has been shown as an important stress-moderating factor ${ }^{24}$ promoting confidence in one's ability to cope with stress and an internally oriented locus of control. ${ }^{25-28}$ Moreover, in line with the cumulative stress hypothesis, ${ }^{29}$ a continuous activation of the stress response systems, over time, would increase the individual's vulnerability to stress-related pathologies ${ }^{30,31}$ and the stress effects on cognitive processes. ${ }^{32,33}$

Stressors, such as the physical and psychological problems related to the caring for a child with chronic respiratory disease, may activate an excessive production of glucocorticoids and a deregulation of cortisol release. Given its high density of glucocorticoid receptors, the hippocampus appears as a structure particularly involved in stress responses and in cumulative exposure to high levels of cortisol ${ }^{34}$ that could have lasting effects on cognitive processes over time. ${ }^{32,33,35}$

Self-esteem shows how the individual considers himself, whereas locus of control refers to the belief of being able to control one's behavior (internal locus of control) or to attribute one's own outcomes to external factors, fatality or chance (external locus of control). Low self-esteem and external control can lead to feelings of helplessness and increasing vulnerability to stress and disease. ${ }^{26}$ In healthy elderly subjects, low self-esteem and low internal locus of control would be associated with smaller hippocampal volume and signs of cortisol deregulation. ${ }^{33}$ Subjects with low self-esteem and low internal locus of control showed a significant cortisol reaction ${ }^{36}$ and continuous high cortisol responses to stress situations. ${ }^{37}$

Conversely, good self-esteem promotes greater confidence in one's ability to cope with stress and a more internally oriented locus of control. ${ }^{38}$ A positive selfimage can promote successful aging, greater overall health and cognitive functioning, and more effective autonomy and independence. ${ }^{39}$ In neuroimaging studies and volumetric analysis of the medial temporal lobe, self-esteem and locus of control were significantly correlated to hippocampal volumes. ${ }^{40}$ Moreover, an association between chronic stress and reduced hippocampal volume has been reported. ${ }^{33,41,42}$

In this background, the current study aimed to investigate parental stress, self-esteem, locus of control and memory performance in a group of mothers of children affected by OSAS for at least 4 years, compared to a group of mothers of typically developing children (TDC). In literature, the correlations between stress, self-esteem, and locus of control and between stress and memory have been widely documented..$^{33,35,40,43}$ The current study novelty is that it refers to issues that are important for therapeutic practice dealing with a very particular stress condition: stress management and memory functioning in mothers of OSAS children. The hypothesis was that OSAS mothers would be more stressed than the TDC mothers and would show a lower self-esteem, an external locus of control and lower memory performance.

\section{Materials and methods}

\section{Study population}

The study sample included 86 mothers (mean age 35.6 \pm 4.9 , ranged between 32 and 41 years) of children diagnosed with OSAS for at least 4 years and referred to the Chair of Child Neuropsychiatry at University of Palermo,

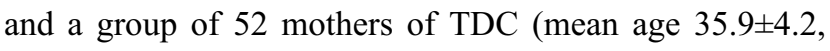
ranged between 32 and 41 years). All participants were from the same urban area, Caucasian, Italian speakers, middle socioeconomic status (average monthly remuneration defined "middle" according to the Italian Statistic Institute tables), education between 8 and 18 


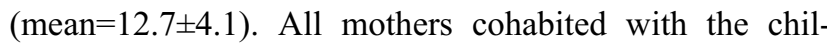
dren's father. Children OSAS diagnosis was made by a trained neuropsychiatry (MC, MR) using the standard criteria for manual visual scoring to identify respiratory and desaturation events. ${ }^{44-46}$

All participants underwent a level of stress evaluation, and, in addition, a global self-esteem, internal/external locus of control and memory assessment. All participants provided a written informed consent. The Departmental Ethics Committee at the Second University of Naples approved the study.

\section{Psychological and memory assessment}

To assess mothers' stress, the Italian version of the Parenting Stress Index-Short Form (PSI-SF) was used. ${ }^{47}$ PSI-SF is a self-rating scale for screening the stress in the parent-child relationship. It consists of three subscales, 12 items for each, measuring three different domains: 1) stress related to parenting role; 2) parent-child dysfunctional interaction; 3) difficult child. 1) The subscale "stress related to the parental role" refers to the stress that parents experience as parents, such as of impaired parental abilities ("I feel trapped by my responsibilities as a parent"), relational difficulties with the partner ("having a child has caused more problems than I expected with partner"), poor social support ("I feel alone and without friends"), and depression ("there are quite a few things that bother me about my life"). 2) "Parent-child dysfunctional interaction" subscale refers to the parent's perception of the child as reinforcing or unresponsive to his expectations: "my child rarely does things for me that make me feel good", "my child smiles at me much less than I expected", "my child is not able to do as much as I expected". 3) "Difficult child" subscale refers to the child's behavioral patterns that make him non-compliant and difficult to manage: "My child seems to cry or fuss more than other children" "my child generally wakes up in a bad mood" "my child's sleeping or eating schedule was much harder to establish that I expected". Mothers were asked to rate each item on a 1 (strongly disagree) to 5 (strongly agree) scale. The scores for each subscale range from 12 to 60 , and the total score at the PSI-SF is given by the sum of the scores obtained in the three subscales. The higher the score, the greater the level of parental stress. Psychometric evidence supports PSI-SF reliability and validity. Internal reliability for the total stress score was
0.92 (Cronbach's alpha). Reliability values for the individual subscales ranged from 0.80 to 0.87 . Test-retest reliability was 0.84 for total stress score and ranged from 0.68 to 0.84 for the three subscales. ${ }^{47}$ The PSI-SF has been widely used, and its psychometric properties have been reported in parents of children with several chronic medical conditions, including a high-risk sample of mothers and their infants. ${ }^{48}$

The Rosenberg Self-esteem Scale was administered to evaluate global self-esteem as general feelings of selfworth and self-acceptance. ${ }^{49}$ The scale consists of 10 items. For each statement, the subject was invited to express agreement, full agreement, disagreement, full disagreement. Each answer is given a score from 0 to 3 . The scale ranges from 0 to 30 scores. The lower the score, the lower the level of self-esteem.

Rotter's Locus of Control Scale ${ }^{50}$ was administered to assess internal and external locus of control. It shows the perception that one's outcomes may be determined by one's actions or are controlled by external factors. The scale is a 29-item forced-choice test, including six filler items. The subject was asked to select the statement that he agrees with the most. One point was scored when a statement showing an external locus of control was selected. The higher the score, the more external the locus of control.

Memory was assessed using Digit span and, Corsi Block Tapping Test ${ }^{51,52}$ assessing verbal and non-verbal short-term memory. Recognition memory was assessed using the recognition memory test (RMT) for words, faces, and buildings. ${ }^{53}$ In the Digit Span and Block, Tapping Test participants required repeating strings of numbers or blocks of increasing length. The total score was the longest sequence of digits or blocks the participant was able to repeat forward (short-term memory) or backward (working memory). RMT is a set of three forcedchoice recognition tasks, matched for difficulty, providing assessment for verbal (words), non-verbal (faces), and topographical (buildings) recognition memory. Each of the three tests contains 30 items. The total score was the sum of the correctly recognized items.

\section{Statistical analysis}

The mean scores for PSI-SF total score and its subscales, global self-esteem measurements, internal/external locus of control scales, and memory assessment, were computed. The Independent samples $t$-test was applied to compare the psychological and neuropsychological 
parameters between the two groups of mothers. In addition, the Cohen's d was also computed to describe the strength of the effect sizes. ${ }^{54} \mathrm{~A} p$-value $\leq 0.05$ was considered to be statistically significant for correlation analyses. All data were coded and analyzed using the commercially available STATISTICA package for Windows (v 6.0; StatSoft Inc, Tulsa, OK, USA).

\section{Results}

When assessing parental stress, OSAS mothers scored higher on the total stress scale and its three subscales compared to TDC mothers. Differences were statistically significant in the Total Stress score, in the parent-child Interaction and in the Difficult Child subscales' scores, strengthened by an effect size between medium and large in all three indices (Table 1). Additionally, OSAS mothers scored significantly lower on the Rosenberg Self-esteem scale and significantly higher on the external locus of control of the Rotter's Locus of Control scale, with a very large effect size in both the scales (Table 1).

Table 2 shows means and standard deviations comparison between the two groups of mothers in memory assessment. OSAS mothers scored lower compared to TDC mothers on both short-term memory and RMTs administered. Significant differences between the two groups of mothers in the digit span memory test forward and in the Corsi block tapping test forward were found, with a medium effect size. In addition, in the backward trials of digit span memory test and the Corsi block tapping test, the differences were also significant with a medium effect size. The two groups of mothers showed significantly different performance in the RMT in all three modalities: words recognition, faces recognition, and buildings recognition. The effect sizes were medium in all three RMTs.

\section{Discussion}

This study investigated parental stress, self-esteem, locus of control and memory performances in a group of mothers of children affected by OSAS, for at least 4 years, compared to a group of mothers of TDC.

OSAS mother's group appeared more stressed than mothers of typical developing children. According to the methodology of the PSI-SF, parenting stress would be related to the parent's response to the parental role as such, the characteristics of the child, and the individual parent-child interaction. ${ }^{47}$ In the current study, the differences between the two groups were significantly higher in the total stress scores and in the two subscales that highlight problematic child's behavior, and the parent-child interaction. Whereas, in the parental distress subscale, the differences between the two groups were lower. What OSAS mothers perceived as a major source of stress is to be a mother of difficult child, than to be a mother as such (eg, "my child is not able to do as much as I expected" or "my child smiles at me much less than I expected"). Mothers of OSAS children seem significantly more disappointed, dissatisfied by the critical aspects of a problematic child, than TDC mothers ("my child turned out to be a bigger problem than I expected" or "my child asks me more than most children"). The OSAS type respiratory disorder is characterized by sudden and unpredictable crises and by a series of numerous comorbidities associated with breathing difficulties and sleep disturbances. Therefore, as expected, its management may become a significant source of stress for the family, especially for the mother as a primary caregiver. These findings are consistent with the hypothesis that the management of a problematic child can be a significant stressor rather than the condition of mother as such, in line with literature data documenting stress in parents with problematic, disabled, autistic children. ${ }^{1,15,16,18,55}$

Table I Means (M) and standard deviations (SD) comparison between the two groups of mothers in the Parenting Stress Index-Short Form (PSI-SF) and its subscales, in the Rosenberg Self-Esteem Scale (RSE) and in the Rotter's Locus of Control Scale (RLC)

\begin{tabular}{|l|l|l|l|l|l|l|l|}
\hline \multirow{2}{*}{ Scale } & \multicolumn{2}{l|}{ OSAS mothers } & \multicolumn{2}{l|}{ TDC mothers } & $\mathbf{t}$ & \multirow{2}{*}{ Cohen's d } \\
\cline { 2 - 7 } & $\mathbf{M}$ & SD & $\mathbf{M}$ & SD & $\mathbf{( d f = 1 3 6 )}$ & \\
\hline Stress total score & 81.24 & 22.82 & 66.11 & 21.87 & 3.83 & 0.002 & 0.68 \\
$\quad$ Parental stress & 22.99 & 12.02 & 22.44 & 11.45 & 0.26 & 0.791 & 0.05 \\
Parent-child interaction & 26.95 & 14.14 & 20.68 & 12.19 & 2.65 & 0.009 & 0.47 \\
Difficult child & 31.94 & 11.31 & 23.59 & 11.02 & 4.24 & 0.001 & 0.74 \\
\hline Self-esteem & 14.28 & 5.24 & 20.32 & 2.99 & 7.59 & 0.001 & 1.41 \\
Locus of control & 22.07 & 4.58 & 16.86 & 3.86 & 6.86 & 0.001 & 1.23 \\
\hline
\end{tabular}

Abbreviations: OSAS, Obstructive Sleep Apnea Syndrome; TDC, typically developing children. 
Table 2 Means (M) and standard deviations (SD) comparison between the two groups of mothers in memory and working memory (WM) performances

\begin{tabular}{|l|l|l|l|l|l|l|l|}
\hline \multirow{2}{*}{ Test } & \multicolumn{2}{l|}{ OSAS mothers } & \multicolumn{2}{l|}{ TDC mothers } & $\mathbf{t}$ & \multirow{2}{*}{ Cohen's d } \\
\cline { 2 - 6 } & $\mathbf{M}$ & SD & $\mathbf{M}$ & $\mathbf{S D}$ & $\mathbf{( d f = 1 3 6 )}$ & \\
\hline Verbal short-term & 6.25 & 0.75 & 6.75 & 0.95 & 3.43 & 0.001 & 0.58 \\
Non-verbal short-term & 6.25 & 0.73 & 6.80 & 1.02 & 3.68 & 0.001 & 0.62 \\
Verbal WM & 4.99 & 0.59 & 5.34 & 0.68 & 3.19 & 0.002 & 0.55 \\
Non-verbal WM & 4.82 & 0.61 & 5.22 & 0.65 & 3.64 & 0.001 & 0.63 \\
Words recognition & 23.70 & 2.38 & 24.73 & 2.54 & 2.40 & 0.017 & 0.42 \\
Faces recognition & 23.31 & 2.20 & 24.75 & 2.34 & 3.21 & 0.002 & 0.63 \\
Buildings recognition & 23.55 & 1.91 & 24.82 & 2.21 & 3.08 & 0.002 & 0.54 \\
\hline
\end{tabular}

Abbreviations: OSAS, Obstructive Sleep Apnea Syndrome; TDC, typically developing children.

Several studies have shown a relationship between stress, behavior, emotion, and cognition. ${ }^{35,56-58}$ A recent study associated low self-esteem, a predictor of greater stress reactivity, ${ }^{40}$ to reduced hippocampal volume. ${ }^{33}$ In the current study, as expected, OSAS mothers showed a lower self-esteem level and a more externalized locus of control, whereas mothers of TDC showed higher selfesteem and a lower external locus of control. Self-esteem and locus of control may be a crucial expression of "selfefficacy" defined as "people's beliefs about their capabilities to exercise control over events that control their lives". 59 A low self-esteem leads to an affective state that does not allow recovering internal resources consistent with the complexity of everyday life, ${ }^{43}$ and may have effects both on behavior and cognition. ${ }^{33,55}$ High stress and low self-esteem do not allow to focus on one's own abilities to coping and solving daily problems. This finding is consistent with studies providing evidences that parents of children with intellectual disabilities or personality and behavioral problems showed higher parenting stress and an external control, ${ }^{18,21,60}$ according to the cognitive model of stress and coping of parents in the families of children with disabilities. ${ }^{61}$

In addition, in the neurocognitive domain, OSAS mother showed lower memory performance than TDC mothers. Both in verbal and non-verbal short term and working memory and in recognition, verbal, non-verbal, and topographical memory, worse performances were found in mothers of OSAS children. It can be hypothesized that the continuous and unpredictable nocturnal respiratory crises, the sleep deprivation and the persistent hypervigilance may be very significant stressors for the OSAS mothers. Therefore, OSAS mothers' poorer memory performance may be considered as a consequence of a persistent exposure to stress hormones, in agreement with several human and animal studies showing that uncontrollable stress causes a complex neuroendocrine response and it has an impact on the brain structures involved in attention maintenance, and in many memory tasks, such as working memory and memory retrieval. ${ }^{62,63}$

In clinical practice, it should be highlighted that the effect of persistent stress of OSAS mothers could have long-term negative consequences on the organization of the brain that will emerge later in life, ${ }^{35,55}$ supporting the need for psychological training to help mothers to manage the stressful event.

Some limitations of this study need to be highlighted. The first limitation was to have only investigated the performance of the mothers and not to have included investigations on the fathers. Further research, including measuring fathers' performance, should extend observations on how stress is managed by the whole family. Second limitation of the study was to use a generic parental stress scale that is not specific to evaluate the impact of respiratory pathologies and its implications on the mother's stress reaction. Although, the parental stress scale proposed can probably recall in mothers the physical and psychological difficulties related to the child's disease (disturbed sleep, anxiety, fears, helplessness), more specific assessment tools could provide more focused evidences.

In sum, further research is needed for a broader understanding of the biopsychological and neuropsychological mechanisms related to the parental stress and neurocognitive performance in mothers of OSAS children. Data suggested the need for targeted psychological support programs for mothers of children with such complex respiratory pathology. These suggestions are consistent 
with studies showing that mothers of children with sleep or behavioral disorders have shown a more internal locus of control after successfully completed a parent-training program. $^{21,64}$

\section{Disclosure}

The authors declare that this research was conducted in the absence of any commercial or financial relationships that could be construed as a potential conflict of interest. The Department of Psychology, Educational Science and Human Movement of the University of Palermo (Italy) supported this Publication Processing.

\section{References}

1. Carotenuto M, Esposito M, Di Pasquale F, De Stefano S, Santamaria F. Psychological, cognitive and maternal stress assessment in children with primary ciliary dyskinesia. World J Pediatr. 2013;4:312-317. doi:10.1007/s12519-013-0441-1

2. Marcus CL, Brooks LJ, Draper KA, et al. Diagnosis and management of childhood obstructive sleep apnea syndrome. Pediatrics. 2012;130:576-584. doi:10.1542/peds.2012-1671

3. Blechner M, Williamson A. Consequences of obstructive sleep apnea in children. Curr Probl Pediatr Adolesc Health Care. 2016;46:19-26. doi:10.1016/j.cppeds.2015.10.007

4. Brunetti L, Francavilla R, Scicchitano P, et al. Impact of sleep respiratory disorders on endothelial function in children. Sci World J. 2013;e719456. doi:10.1155/2013/719456

5. Carotenuto M, Esposito M, Parisi L, et al. Depressive symptoms and childhood sleep apnea syndrome. Neuropsychiatr Dis Treat. 2012;8:369-373. doi:10.2147/NDT.S35974

6. Landau YE, Bar-Yishay O, Greenberg-Dotan S, Goldbart AD, Tarasiuk A, Tal A. Impaired behavioral and neurocognitive function in preschool children with obstructive sleep apnea. Pediatr Pulmonol. 2012;47(2):180-188. doi:10.1002/ppul.21534

7. Esposito M, Antinolfi L, Gallai B, et al. Executive dysfunction in children affected by obstructive sleep apnea syndrome: an observational study. Neuropsychiatr Dis Treat. 2013;9:1087-1094. doi:10.2147/NDT.S47287

8. Esposito M, Verrotti A, Gimigliano F, et al. Motor coordination impairment and migraine in children: a new comorbidity? Eur $J$ Pediatr. 2012;171(11):1599-1604. doi:10.1007/s00431-012-1759-8

9. Jackman AR, Biggs SN, Walter LM, et al. Sleep-disordered breathing in preschool children is associated with behavioral, but not cognitive, impairments. Sleep Med. 2012;13(6):621-631. doi:10.1016/j.sleep.2012.01.013

10. Smirni D, Oliveri M, Turriziani P, Di Martino G, Smirni P. Benton visual form discrimination test in healthy children: normative data and qualitative analysis. Neurol Sci. 2018;39(5):885-892. doi:10.1007/s10072-018-3297-2

11. Halbower AC, Degaonkar M, Barker PB, et al. Childhood obstructive sleep apnea associates with neuropsychological deficits and neuronal brain injury. PLoS Med. 2006;3(8):e301. doi:10.1371/journal.pmed.0030301

12. Baldassari CM, Alam L, Vigilar M, Benke J, Martin C, Ishman S. Correlation between REM AHI and quality-of-life scores in children with sleep-disordered breathing. Otolaryngol Head Neck Surg. 2014;151(4):687-691. doi:10.1177/0194599814547504

13. Iavarone A, Ziello AR, Pastore F, Fasanaro AM, Poderico C. Caregiver burden and coping strategies in caregivers of patients with Alzheimer's disease. Neuropsychiatr Dis Treat. 2014;29 (10):1407-1413. doi:10.2147/NDT.S58063
14. Santamaria F, Esposito M, Montella S, et al. Sleep disordered breathing and airway disease in primary ciliary dyskinesia. Respirology. 2014;19(4):570-575. doi:10.1111/resp.12273

15. Altiere MJ, von Kluge S. Family functioning and coping behaviors in parents of children with autism. J Child Fam Stud. 2009;18:83-92. doi:10.1007/s10826-008-9209-y

16. Smith LE, Seltzer MM, Tager-flusberg H, Greenberg JS, Carter AS. A comparative analysis of well-being and coping among mothers of toddlers and mothers of adolescents with ASD. J Autism Dev Disord. 2008;38:876-889. doi:10.1007/s10803-007-0461-6

17. Dabrowska A, Pisula E. Parenting stress and coping styles in mothers and fathers of pre-school children with autism and Down syndrome. $J$ Intellect Disabil Res. 2010;54(3):266-280. doi:10.1111/j.13652788.2010.01258.x

18. Hassall R, Rose J, McDonald J. Parenting stress in mothers of children with an intellectual disability: the effects of parental cognitions in relation to child characteristics and family support. $J$ Intellect Disabil Res. 2005;49:405-418. doi:10.1111/j.1365-2788.2005.00673

19. Spratt EG, Saylor CF, Macias MM. Assessing parenting stress in multiple samples of children with special needs (CSN). Families Systems Health. 2007;25:435-449.

20. Tomanik S, Harris GE, Hawkins J. The relationship between behaviour exhibited by children with autism and maternal stress. $J$ Intellect Dev Disabil. 2004;29:16-26. doi:10.1080/13668250410001662892

21. Wiggs L, Stores G. Behavioural treatment for sleep problems in children with severe intellectual disabilities and daytime challenging behaviour: effect on mothers and fathers. Br J Health Psychol. 2001;6:257-269. doi:10.1348/135910701169197

22. Abramson LY, Seligman MEP, Teasdale JD. Learned Helplessness in Humans: critique and Reformulation. Engl J Abnorm Psychol. 1978;87(1):49-74. doi:10.1037/0021-843X.87.1.49

23. Technow JR, Hazel NA, Abela JRZ, Hankin BL. Stress sensitivity interacts with depression history to predict depressive symptoms among youth: prospective changes following first depression onset. J Abnorm Child Psychol. 2015;43(3):489-501. doi:10.1007/s10802014-9922-5

24. Carson J, Kuipers E. Stress management interventions. In: Hardy S, Carson J, Thomas B, editors. Occupational Stress: Personal and Professional Approaches. Cheltenham: Stanley Thornes; 1998:157-174.

25. DeLongis A, Folkman S, Lazarus RS. The impact of daily stress on health and mood: psychological and social resources as mediators. $J$ Pers Soc Psychol. 1988;54:486-495.

26. Leary MR, MacDonald G. Individual differences in trait self-esteem: a theoretical integration. In: Leary M, Tangney J, editors. Handbook of Self and Identity. New York: Guilford Press; 2003:401-418.

27. Lo R. A longitudinal study of perceived level of stress, coping and self-esteem on undergraduate nursing students: an Australian case study. J Adv Nurs. 2002;39:119-126.

28. Whisman MA, Kwon P. Life stress and dysphoria: the role of self esteem and hopelessness. J Pers Soc Psychol. 1993;65:1054-1060.

29. Nederhof E, Schmidt MV. Mismatch or cumulative stress: toward an integrated hypothesis of programming effects. Physiol Behav. 2012;106(5):691-700. doi:10.1016/j.physbeh.2011.12.008

30. McEwen BS. Stress, adaptation, and disease. Allostasis andallostatic load. Ann N Y Acad Sci. 1998;840:33-44.

31. McEwen BS. Physiology and neurobiology of stress and adaptation: central role of the brain. Physiol Rev. 2007;87(3):873-904. doi:10.1152/physrev.00041.2006

32. Forns J, Vegas O, Julvez J, et al. Association between child cortisol levels in saliva and neuropsychological development during the second year of life. Stress Health. 2014;30(2):142-148. doi:10.1002/ smi.2504

33. Pruessner JC, Baldwin MW, Dedovic K, et al. Self-esteem, locus of control, hippocampal volume, and cortisol regulation in young and old adulthood. Neuroimage. 2005;28:815-826. doi:10.1016/j. neuroimage.2005.06.014 
34. Fuchs E, Flugge G. Chronic social stress: effects on limbic brain structures. Physiol Behav. 2003;79:417-427.

35. Lupien SJ, Fiocco A, Wan N, et al. Stress hormones and human memory function across the lifespan. Psychoneuroendocrinology. 2005;30:225-242. doi:10.1016/j.psyneuen.2004.08.003

36. Pruessner JC, Hellhammer DH, Kirschbaum C. Burnout, perceived stress, and cortisol responses to awakening. Psychosom Med. 1999;61:197-204.

37. Kirschbaum C, Prussner JC, Stone AA. Persistent high cortisol responses to repeated psychological stress in a subpopulation of healthy men. Psychosom Med. 1995;57:468-474.

38. Baumeister RF, Campbell JK, Krueger JI, Vohs KD. Does high selfesteem cause better performance, interpersonal success, happiness, or healthier lifestyles? Psychol Sci Public Interest. 2003;4:1-44. doi:10.1111/1529-1006.01431

39. Markus H, Herzog R. The role of the self-concept in aging. Annu Rev Gerontol Geriatr. 1991;11:111-143.

40. Pruessner JC, Lord C, Meaney M, Lupien SJ. Effects of self-esteem on age-related changes in cognition and the regulation of the hypothalamic-pituitary-adrenal axis. Ann NY Acad Sci. 2004;1032:186-194. doi:10.1196/annals.1314.017

41. Chan SW, Harmer CJ, Norbury R, O’Sullivan U, Goodwin GM, Portella MJ. Hippocampal volume in vulnerability and resilience to depression. $J$ Affect Disord. 2016;189:199-202. doi:10.1016/j.jad.2015.09.021

42. Roddy DW, Farrell C, Doolin K, et al. The hippocampus in depression: more than the sum of its parts? Advanced hippocampal substructure segmentation in depression. Biol Psychiatry. 2018;pii: S0006-3223(18)31819-5. doi:10.1016/j.biopsych.2018.08.021

43. Tafarodi RW, Marshall TC, Milne AB. Self-esteem and memory. $J$ Pers Soc Psychol. 2003;84(1):29-45. doi:10.1037/0022-3514.84.1.29

44. Berry RB, Budhiraja R, Gottlieb DJ, et al. American Academy of Sleep Medicine. Rules for scoring respiratory events in sleep: update of the 2007 AASM manual for the scoring of sleep and associated events. Deliberations of the sleep apnea definitions task force of the American Academy of Sleep Medicine. J Clin Sleep Med. 2012;8 (5):597-619. doi:10.5664/jcsm.2172

45. Goh DY, Galster P, Marcus CL. Sleep architecture and respiratory disturbances in children with obstructive sleep apnea. Am J Respir Crit Care Med. 2000;162:682-686. doi:10.1164/ajrccm.162.2.9908058

46. Wagner MH, Torrez DM. Interpretation of the polysomnogram in children. Otolaryngol Clin North Am. 2007;40(4):745-759. doi:10.1016/j. otc.2007.04.004

47. Abidin RR. Parenting Stress Index. 3rd ed. Odessa, FL: PAR; 1995.

48. Barroso N, Hungerford GM, Garcia D, Graziano PA, Bagner DM. Psychometric properties of the Parenting Stress Index-Short Form (PSI-SF) in a high-risk sample of mothers and their infants. Psychol Assess. 2016;28(10):1331-1335. doi:10.1037/pas0000257

49. Rosenberg M. Society and the Adolescent Self-Image. Princeton, NJ: Princeton University Press; 1965.
50. Rotter JB. Generalized expectancies for internal versus external control of reinforcement. Psychol Monogr. 1966;80:1-28. Whole No. 609. doi: $10.1037 / \mathrm{h} 0092976$

51. Monaco M, Costa A, Caltagirone C, Carlesimo GA. Forward and backward span for verbal and visuo-spatial data: standardization and normative data from an Italian adult population. Neurol Sci. 2013;34:749-754. doi:10.1007/s10072-012-1130-x

52. Orsini A, Grossi D, Capitani E, Laiacona M, Papagno C, Vallar G. Verbal and spatial immediate memory span: normative data from 1355 adults and 1112 children. Ital $J$ Neurol Sci. 1987;8:539-548.

53. Smirni D, Smirni P, Di Martino G, Cipolotti L, Oliveri M, Turriziani P. Standardization and validation of a parallel form of the verbal and non verbal recognition memory test in an Italian population sample. Neurol Sci. 2018;39(8):1391-1399. doi:10.1007/s10072-018-3433-z

54. Cohen J. Statistical Power Analysis for the Behavioral Sciences (2. Auflage). Hillsdale, NJ: Erlbaum; 1998.

55. Lupien SJ, McEwen BS, Gunnar MR, Heim C. Effects of stress throughout the lifespan on the brain, behavior and cognition. Nat Rev Neurosci. 2009;10:434-445. doi:10.1038/nrn2639

56. Lupien SJ, McEwen BS. The acute effects of corticosteroids on cognition: integration of animal and human model studies. Brain Res Brain Res Rev. 1997;24:1-27.

57. Smirni D, Beadle JN, Paradiso S. An initial study of alexithymia and its relationship with cognitive abilities among mild cognitive impairment, Mild Alzheimer's disease, and healthy volunteers. J Nerv Ment Dis. 2018;206(8):628-636. doi:10.1097/NMD.0000000000000853

58. Smirni D, Smirni P, Di Martino G, Operto FF, Carotenuto M. Emotional awareness and cognitive performance in borderline intellectual functioning young adolescents. J Nerv Ment Dis. 2019;207 (5):365-370. doi:10.1097/NMD.0000000000000972

59. Bandura A. Self-efficacy mechanism in human agency. Am Psychol. 1982;37:122-147. doi:10.1037/0003-066X.37.2.122

60. Hagekull B, Bohlin G, Hammarberg A. The role of parental perceived control in child development: a longitudinal study. Int J Behav Dev. 2001;25:429-437. doi:10.1080/016502501316934851

61. Lazarus RS, Folkman S. Stress, Appraisal and Coping. New York: Springer; 1984.

62. Kim EJ, Pellman B, Kim JJ. Stress effects on the hippocampus: a critical review. Learn Mem. 2015;22(9):411-416. doi:10.1101/lm.037291.114

63. Wolf OT, Atsak P, de Quervain DJ, Roozendaal B, Wingenfeld K. Stress and memory: a selective review on recent developments in the understanding of stress hormone effects on memory and their clinical relevance. J Neuroendocrinol. 2016;28:8. doi:10.1111/ jne. 12353

64. Roberts MW, Joe VC, Rowe-Hallbert A. Oppositional child behavior and parental locus of control. J Clin Child Adolesc Psychol. 2001;21:170-177. doi:10.1207/s15374424jccp2102_9
Psychology Research and Behavior Management is an international, peer-reviewed, open access journal focusing on the science of psychology and its application in behavior management to develop improved outcomes in the clinical, educational, sports and business arenas. Specific topics covered in the journal include: Neuroscience, memory and decision making; Behavior modification and management; Clinical applications; Business and sports performance management; Social and developmental studies; Animal studies. The manuscript management system is completely online and includes a very quick and fair peer-review system, which is all easy to use. Visit http://www. dovepress.com/testimonials.php to read real quotes from published authors. 